$\xi=-$ 圆

\title{
A Multiscale Fusion Approach for Change Detection in SAR Images
}

\author{
R. Vijayageetha ${ }^{1}$, S. Kalaivani ${ }^{2} *$ \\ ${ }^{1}$ Research Scholar, School of Electronics Engineering, VIT University, Vellore \\ ${ }^{2}$ Associate Professor, School of Electronics Engineering, VIT University, Vellore \\ *Corresponding author E-mail: kalaivani.s@vit.ac.in
}

\begin{abstract}
Best performance and greatness in precise changes are vital factors of change detection. The proposed method is mutual task to deal about preprocessing and change detection of multitemporal SAR images. In preprocessing stage, Speckle Reducing Anisotropic Diffusion is implemented in each layer of multiscale pyramid transform. The speckle free images are interpreted by Absolute difference method and XOR operator to retrieve primary difference image. After that desired change detection is fused by laplacian pyramid coefficient. Fused difference image incorporates the advantages of absolute difference and XOR operation. Finally robotic threshold algorithm of Otsu is used to predict exact change detection. For experimental purposes two data sets are preferred from Envisat and TerraSAR-X images. Standard quality has been evaluated on the proposed method to quantitatively prove the performance.
\end{abstract}

Keywords: Speckle, Difference image, Laplacian pyramid fusion, Otsu threshold algorithm, Performance evaluation, SAR image.

\section{Introduction}

The Change Detection (CD) is the ultimate task of remote sensing to discover the differences between two same geographical images at specific times. This geological image is carried out by microwave sensors of Synthetic Aperture Radar (SAR) images [1]. Microwave sensors are active sensors which are not conscious of climate and illumination conditions. It can operate with all occasions; all weather and vast area coverage. Therefore, active sensors are more dynamic change prediction than passive sensors. However, SAR images are plagued by multiplicative noise described as speckle. Speckle reduction is not only to reduce noise along with preventing fade away signals and preserve boundaries of the image. The change detection is executed by three primary stages [5] (1) Preprocessing (2) Image comparison (3) Applying appropriate threshold methods for change detection. In the initial stage, speckle noise reduction is a crucial chore to focus on. This will deteriorate the post processing procedures like registration, change analysis, etc. Hence a speckle noise is treated as an influenced source of multiplicative noise in SAR image and consider to process without disturbing the essential image appearances. Speckle reduction techniques have to ensure that should not introduce any undesirable information. To overcome these problems, diffusion based speckle reducing anisotropic diffusion (SRAD) method is introduced [9]. This will weaken the speckle noise and enhance edge information. In second stage, the comparisons of two registered images [10] are executed by the algebraic methods of image difference, absolute difference, change vector analysis, image regression and image ratio operator. The proposed method uses absolute difference method (AD) [3] and Foreground detection (FD) method for identifying the difference images. The absolute difference is defined as pixel by pixel value of magnitude difference between two images. The foreground detection is carried out by logical operation, which is a powerful analysis in im- age processing based on morphology. An XOR operator is performed on a pixel by pixel basis between two images [4]. Then, the interesting idea is acquiring perfect DI by Laplacian pyramid fusion coefficients rather than the individual result of DIs. The change detection usually considered in two classes: changed and unchanged. Hence the multi scale fusion [7] method will retaining background region and enhance the foreground objects. The optimal difference image has less intensity value in unchanged region and high intensity values for changed region. On the final stage, convenient thresholding techniques are employed to distinguish the CD. Threshold technique is very simple and effective tool to separate a grayscale image into a binary image. In this paper, an Otsu's threshold algorithm [6] is preferred to select an automatic threshold value based on gray level distribution.

\section{Methodology}

This paper aims an exclusive method of fusion based change detection in multitemporal SAR images under unsupervised category. This session describes the proposed method for change detection in SAR images. It contains five stages: (1) Preprocessing by LPSRAD (2) Difference image evaluating by Absolute difference (AD) and Foreground detection (FD) methods. (3) Then resultant images of $\mathrm{AD}$ and $\mathrm{FD}$ are fused by the Laplacian pyramid fusion approach. (4) Fused image is applied with the automatic Otsu's threshold algorithm. (5) Finally, to recognize the excellent change detection by quality measures. This paper is an integrity method for preprocessing and change detection. SAR images can perform at all times and all weather conditions; the only impact is speckle reduction of SAR images. The proposed method is illustrated in Fig. 1. 


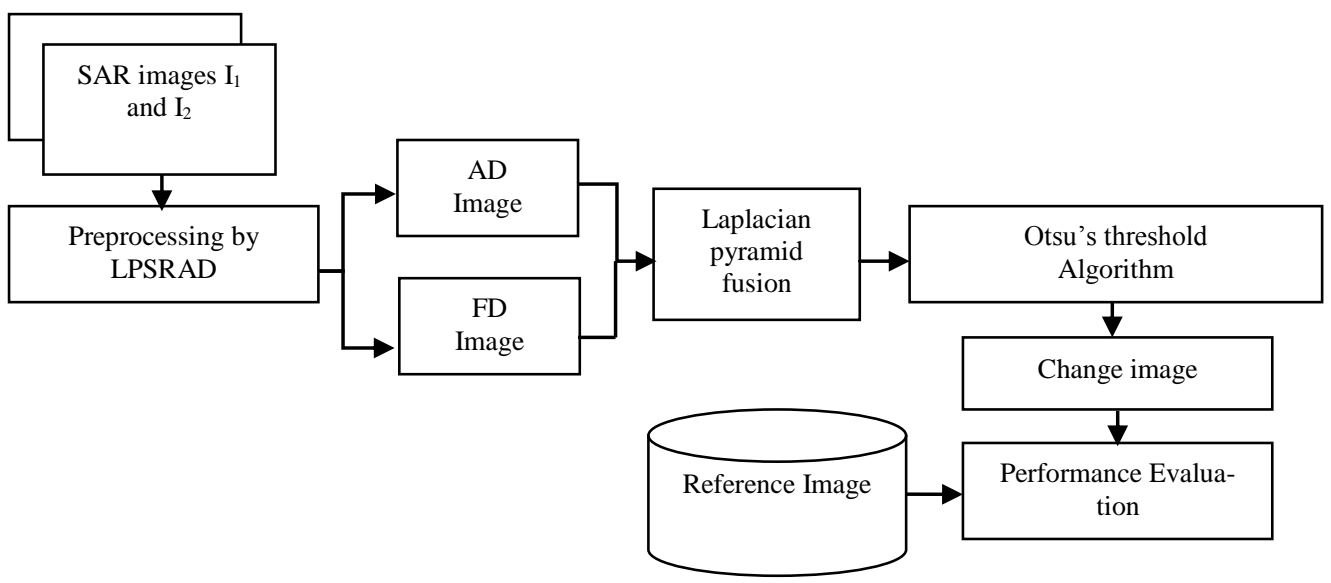

Fig. 1: Block diagram of Fusion based change detection

\subsection{Laplacian Pyramids based Speckle Reducing Aniso- tropic Diffusion}

The construction of image pyramid is a hierarchical structure of multilayer representation. The image pyramid has two stages: decomposition and reconstruction. In the decomposition, the image is decomposed by the approximation filter and reconstructing by interpolation filter. The image pyramid offers two types: Gaussian and Laplacian pyramids [8]. The Laplacian pyramid obeys reduce and Expand operators [9]. In reduce operator curtails an image by the low pass filter subsequently sub sampling by factor of two. The Expand operator magnifies an image twice in size with up sampling followed by low pass filter. The original SAR image I, the Gaussian and Laplacian pyramids are denoted by $\mathrm{G}_{\text {lap }}$ and $\mathrm{L}_{\text {lap }}$. Where lap $=1$ to $\mathrm{d}$ number of decomposition levels. The Gaussian and Laplacian pyramid are given below.

$$
\begin{aligned}
& G_{0}=I \\
& G_{\text {lap }}=\operatorname{reduce}\left[G_{\text {lap }}-1\right] \\
& L_{\text {lap }}=G_{\text {lap }}-\operatorname{Expand}\left[G_{\text {lap }}+1\right]
\end{aligned}
$$

Each Laplacian pyramid layer is manipulating the pyramid coefficients by Speckle reducing anisotropic diffusion (SRAD) [2]. Yu and Acton proposed [10] the desired SRAD for noise removal application is given below,

$$
\left\{\begin{array}{c}
\partial I(x, y ; t) / \partial t=\operatorname{div}[c(q) \nabla I(x, y ; t)] \\
I(x, y ; 0)=I_{0}(x, y),\left(\partial I(x, y ; t) /\left.\partial \vec{n}\right|_{\partial \Omega}=0\right)
\end{array}\right.
$$

where $c(q)$ denotes the diffusion coefficient, $\partial \Omega$ denotes the border of $\Omega, \vec{n}$ is the outer normal to the $\partial \Omega$. The pyramid coefficient is implemented with SRAD, such multilayer approach is termed as Laplacian pyramid based speckle reducing anisotropic diffusion (LPSRAD) [9] method.

\subsection{Difference Image Generation}

Unsupervised change detection is recognized by generating and evaluating the DI on a pixel by pixel comparison. Let consider two SAR images acquired in same geological aspects with time variations are denoted as date 1 and 2 respectively are $I_{1}, I_{2}$.

$$
\begin{aligned}
& l_{1}=I_{\text {date }}^{i, l} \quad \text { where } i=1 \ldots n . \\
& l_{2}=l_{\text {date2 }}^{i, j} \\
& j=1 \ldots m .
\end{aligned}
$$

Where $n, m$ represents rows and columns of images. Numerous CD methods are available to extract perfect changes of multitemporal SAR images. Very simple and less computational complexity methods are proposed in this paper. SAR images are analyzed by an Absolute Difference method and Foreground detection method.

\subsubsection{Absolute Difference Image (AD):}

The Absolute Difference method is a pixel-by-pixel subtraction of two same geographical images. The magnitude difference image will be an absolute value. The maximum intensity value represents changed area and minimum value represents unchanged area. The simple threshold is applied here based on the intensity value of the reference image. The absolute difference is defined below

$A D I=\left|I_{1}-I_{2}\right|$

\subsubsection{Foreground Detection (FD):}

The foreground detection method is implemented by the pixel wise XOR operator. The pixel wise XOR operator offer binary ' 1 ' if just one and only one of the input values is high, and binary ' 0 ' for otherwise. The value ' 1 ' (white) pixels are represents as the changed area. This foreground detection operator is given below

$$
F D I=\left(I_{1} \otimes I_{2}\right)
$$

\subsection{Multiscale Fusion Approach}

The excellency of image fusion is to extract the maximum important information of multiple image of same geographical area that is not possible in individual aspects. Laplacian pyramid is a popular fusion approach to preserve the detail information at each level so that it retains edge information and reduce artifacts. In this proposed method, the primary levels of different images from $A D$ and $F D$ are fused by a Laplacian pyramid (LP) approach. The desirable fused image merges their corresponding important detail and maintains the information as affluent as possible. The Laplacian pyramid approach allows an image to imply fusion rule in each level of pyramid transform. Here, two important fusion rules are used: the rule of maximum local area energy coefficient for levels of band pass images and the standard deviation and entropy merged for an absolute level of the low pass band image. 


$$
\begin{aligned}
& L P_{L L}^{F}= \begin{cases}L_{L}^{A D}(i, j) & S D^{A D}(i, j) \geq S D^{F D}(i, j) \& E^{A D}(i, j) \geq E^{F D}(i, j) \\
L_{L}^{F D}(i, j) & S D^{A D}(i, j)<S D^{F D}(i, j) \& E^{A D}(i, j)<E^{F D}(i, j) \\
\left(L_{L}^{A D}(i, j)+L_{L}^{F D}(i, j)\right) / 2 \text { otherwise }\end{cases} \\
& L P_{\varepsilon}^{F}(i, j)= \begin{cases}L_{\varepsilon}^{A D}(i, j) & e_{\varepsilon}^{A D}(i, j) \geq e_{\varepsilon}^{F D}(i, j) \\
L_{\varepsilon}^{F D}(i, j) & e_{\varepsilon}^{A D}(i, j)<e_{\varepsilon}^{F D}(i, j)\end{cases} \\
& e_{\varepsilon}(i, j)=\sum_{m \in i, n \in j}\left[L_{\varepsilon}(m, n)\right]^{2}
\end{aligned}
$$

Here LL represents a low pass signal of two images. $S D^{A D}(i, j)$, $S D^{F D}(i, j)$ are standard deviation and $E^{A D}(i, j), E^{F D}(i, j)$ are Entropy for corresponding $L_{L}^{A D}(i, j), L_{L}^{F D}(i, j)$ images. The $L P_{\epsilon}(i, j)$ for rest of band pass component at point $(i, j)$ of corresponding image. $\mathrm{e}_{\epsilon}(\mathrm{i}, \mathrm{j})$ represents local area energy coefficient at point $(\mathrm{i}, \mathrm{j})$ and local window $(m, n)$ is centered on point $(i, j)$. The $L_{\epsilon}(m, n)$ denoted

\begin{tabular}{|c|c|c|c|}
\hline Quality measure with description & Envisat dataset & $\begin{array}{c}\text { TerraSAR-X } \\
\text { dataset }\end{array}$ & ERS-2 dataset \\
\hline $\begin{array}{l}\text { ENL } \\
\text { Equivalent number of looks. The amplitude image is calculated by ratio of mean to } \\
\text { variance. Maximum value corresponds to the better speckle reduction. }\end{array}$ & 31.6512 & 42.4006 & 35.4621 \\
\hline $\begin{array}{l}\text { SSI } \\
\text { Speckle Suppression Index. It is defined by the coefficient of variance of speckle free } \\
\text { image normalized by the original image. SSI provides better result for lesser than one. }\end{array}$ & 0.2199 & 0.1802 & 0.2003 \\
\hline $\begin{array}{l}\text { PSNR } \\
\text { Peak signal to noise ratio. It measures the logarithmic value of maximum fluctuations } \\
\text { between input signals to mean square error. }\end{array}$ & 57.8942 & 44.3694 & 49.3253 \\
\hline
\end{tabular}
maximum local area energy coefficient around the local window. After obtaining from the decomposed fused process all levels have to reconstruct to get a desired fuse image.

Table 1: LPSRAD filter evaluation results

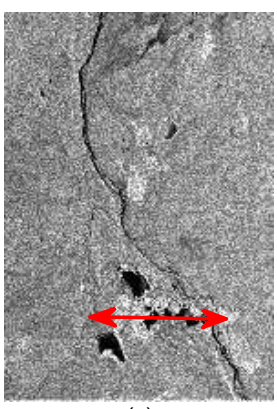

(a)
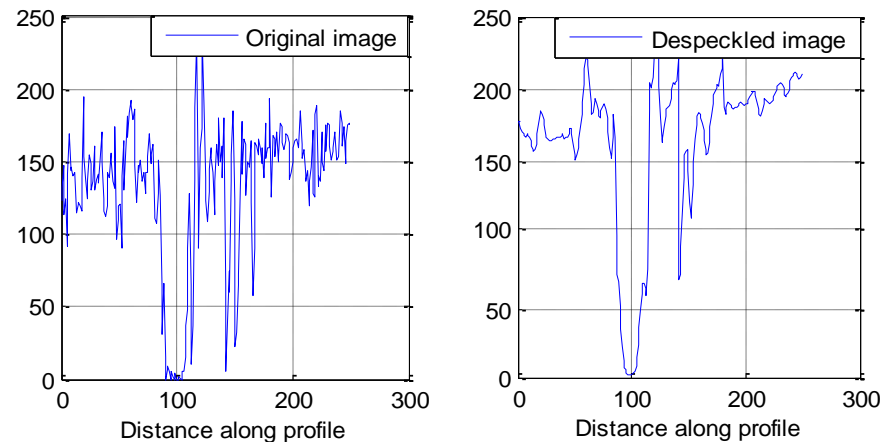

Fig. 2: Experimental results of image profile (a) Envisat image with marginal on $310^{\text {th }}$ row, Distance along profile on Original and despeckled image

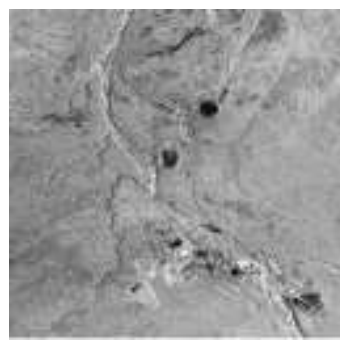

(a)

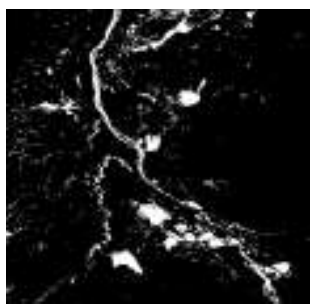

(d)

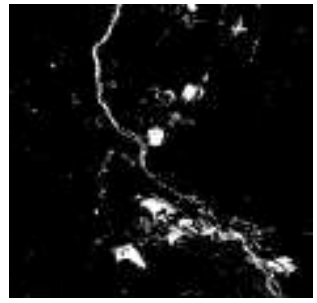

(i)

(e)

(j)

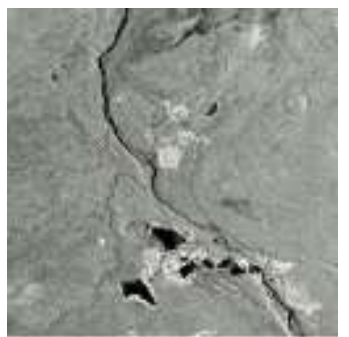

(b)


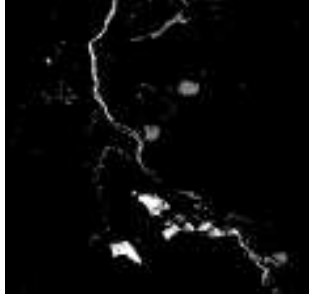

(f)

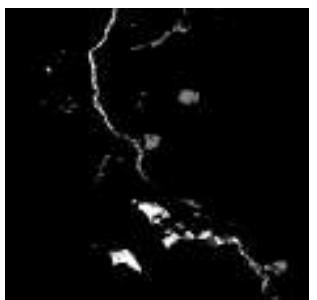

(k)

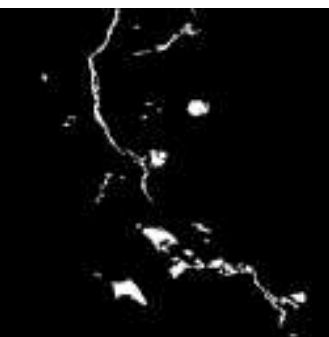

(c)

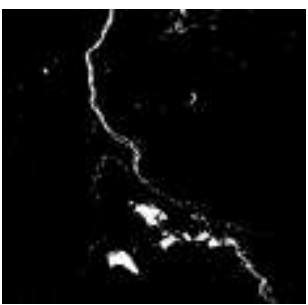

(g)

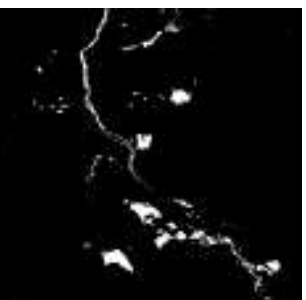

(1)

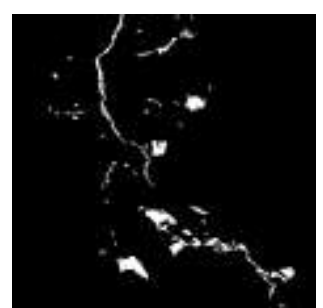

(h)

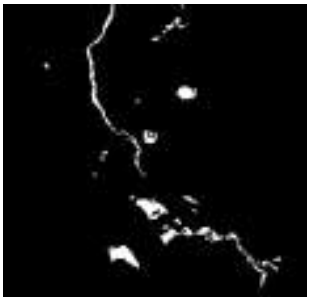

(m)

Fig. 3: Experimental results of Envisat dataset (a) Original image1 (b) Original image2 (c) Ground Truth (d) MR (e) LR (f) WFML (g) WFMLO (h) LPFMLO (i) AD (j) FD (k) WFAX (l) WFAXO (m) LPFAXO 
Table 2: Performance Evaluation for Envisat dataset

\begin{tabular}{|c|c|c|c|c|c|c|c|c|c|c|c|c|}
\hline Methods & $\mathbf{F}_{\mathbf{P}}$ & $\mathbf{F}_{\mathrm{N}}$ & $\mathbf{T}_{\mathbf{P}}$ & $\mathbf{T}_{\mathrm{N}}$ & $\mathbf{P}_{\mathbf{C C}}$ & FAR & $\mathbf{F}_{1}$ & $\mathbf{O}_{\mathrm{E}}$ & $\mathbf{E}_{\mathrm{R}}$ & $\mathbf{K}_{\mathbf{C}}$ & $\mathbf{P}$ & G \\
\hline MR & 5063 & 6185 & 4400 & 105762 & 0.9074 & 0.0457 & 0.4389 & 11248 & 0.0926 & 0.3886 & 0.465 & 0.4396 \\
\hline LR & 2636 & 430 & 2265 & 116079 & 0.9747 & 0.0222 & 0.5964 & 3066 & 0.0253 & 0.5845 & 0.4622 & 0.6232 \\
\hline WFML & 2046 & 45 & 1589 & 117370 & 0.9827 & 0.0171 & 0.6032 & 2091 & 0.0173 & 0.5956 & 0.4371 & 0.652 \\
\hline WFMLO & 1429 & 255 & 2226 & 117155 & 0.9861 & 0.0121 & 0.7256 & 1684 & 0.0139 & 0.7187 & 0.609 & 0.7392 \\
\hline LPMLO & 1026 & 428 & 2242 & 117714 & 0.988 & 0.0086 & 0.7551 & 1454 & 0.012 & 0.7491 & 0.686 & 0.759 \\
\hline ADI & 4100 & 438 & 2213 & 114659 & 0.9626 & 0.0345 & 0.4938 & 4538 & 0.0374 & 0.4777 & 0.3505 & 0.541 \\
\hline FDI & 1911 & 287 & 1654 & 117558 & 0.9819 & 0.016 & 0.6008 & 2198 & 0.0181 & 0.5924 & 0.464 & 0.6288 \\
\hline WFAX & 1932 & 61 & 2113 & 117304 & 0.9836 & 0.0162 & 0.6795 & 1993 & 0.0164 & 0.6719 & 0.5224 & 0.7125 \\
\hline LPAXO & 370 & 430 & 2033 & 118577 & 0.9934 & 0.0031 & 0.8356 & 800 & 0.0066 & 0.8322 & 0.846 & 0.8357 \\
\hline
\end{tabular}

\subsection{Otsu's Thresholding Algorithm}

An image I(x,y) contains spatial resolution of $\mathrm{n} x \mathrm{~m}$ with normalized histogram of gray values varies from 0 to $l-1$ (where $l$ is gray levels, $\mathrm{n}_{\mathrm{i}}$ is number of pixels $\mathrm{i} \in[0, l]$ and $\mathrm{N}$ is total number of pixels) The probability of occurrence $\mathrm{P}_{\mathrm{i}}$ is given below [6],

$$
P_{i}=\frac{n_{i}}{N} \text { where } \quad N=\sum_{i=1}^{L} n_{i}
$$

Consider an image is separated into two classes $C_{1}(k)$ and $C_{2}(k)$ corresponds to background and foreground pixels. $\mathrm{C}_{1}(\mathrm{k})$ consists of pixel levels of $[1,2, \ldots \mathrm{k}]$ and $\mathrm{C}_{2}(\mathrm{k})$ consists of pixels with levels of $[k+1, \ldots .1]$. The probability distribution of $P_{1}(k)$ and $P_{2}(k)$ are given as

$$
P_{1}(k)=\sum_{i=1}^{k} P_{f} \quad \text { and } \quad P_{2}(k)=\sum_{i=k-1}^{t} P_{t}=1-P_{1}(k)
$$

The mean values of $\mathrm{C} 1(\mathrm{k})$ and $\mathrm{C}_{2}(\mathrm{k})$ are denotes by $\mu_{1}(\mathrm{k})$ and $\mu_{2}(k)$. The class variance for background and foreground are, $\sigma_{1}^{2}(\mathrm{k}), \sigma_{2}^{2}(\mathrm{k})$. The class variance can be denoted as withinclass $\sigma_{w}^{2}(\mathrm{k})$ and between class variance $\sigma_{B}^{2}(\mathrm{k})$,

The total mean value of given image is

$\mu_{T}=\sum_{i=1}^{l} i P_{i}=C_{1}(k) \mu_{1}(k)+C_{2}(k) \mu_{2}(k)$

$\sigma_{B}^{2}(k)=P_{1}(k)\left(\mu_{1}(k)-\mu_{T}\right)^{2}+P_{2}(k)\left(\mu_{2}(k)-\mu_{T}\right)^{2}$

$\sigma_{W}^{2}(k)=P_{1}(k) \sigma_{1}^{2}(k)+P_{2}(k) \sigma_{2}^{2}(k)$

The automatic threshold $\mathrm{t}_{\mathrm{n}}$ depends on either maximizing $\sigma_{B}^{2}(\mathrm{k})$ or minimizing $\sigma_{w}^{2}(\mathrm{k})$.

$t_{n}=\arg \max _{0 \leq k \leq l} \sigma_{B}^{2}(k)$



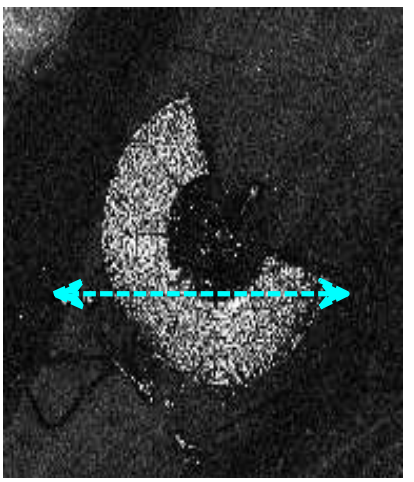

(c)
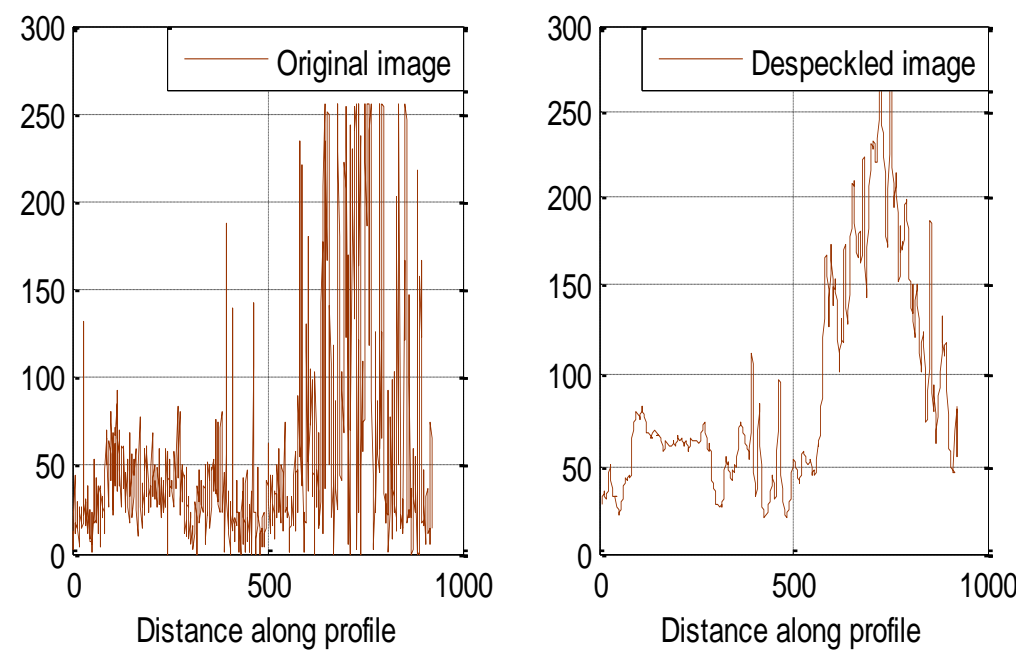

Fig. 4: Experimental results of image profile (c) TerraSAR-X dataset with marginal on $940^{\text {th }}$ row, Distance along profile on Original and despeckled image 
Table 3: Performance Evaluation for TerraSAR-X dataset

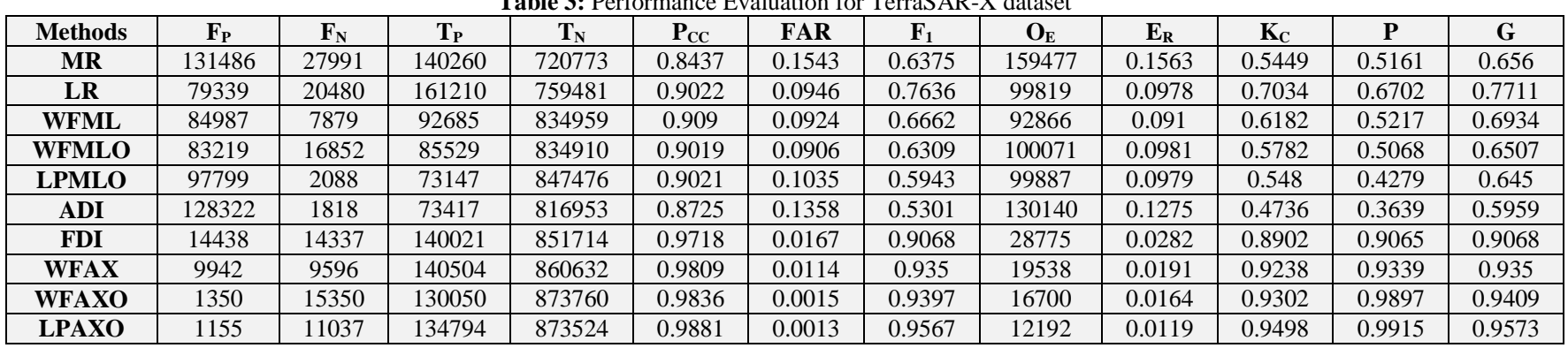

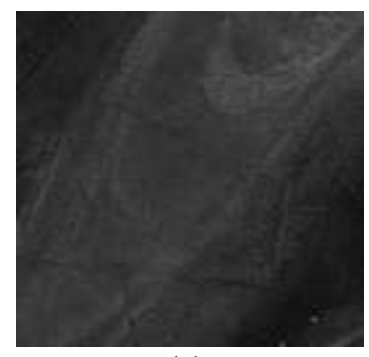

(a)

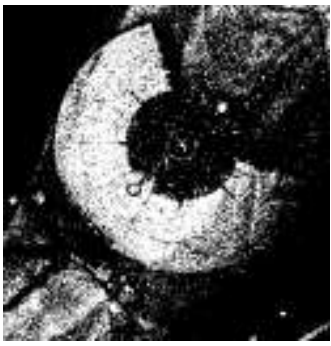

(d)



(i)

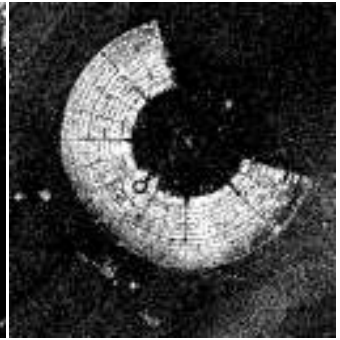

(e)

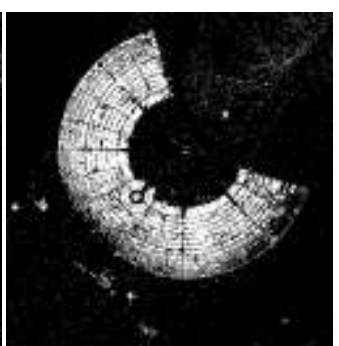

(j)

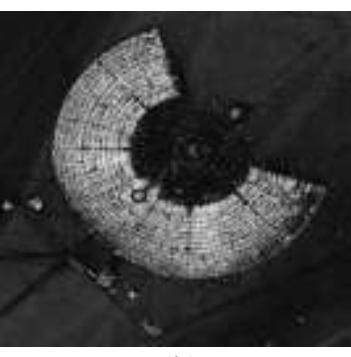

(b)

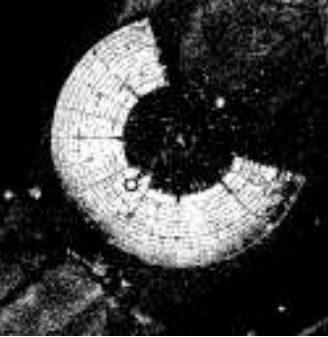

(f)

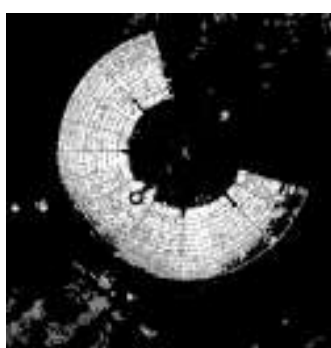

(k)

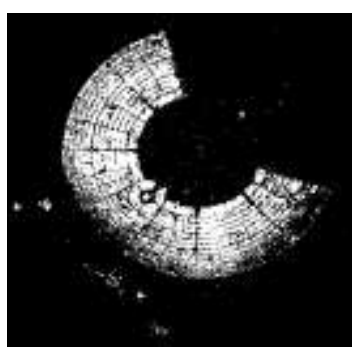

(c)

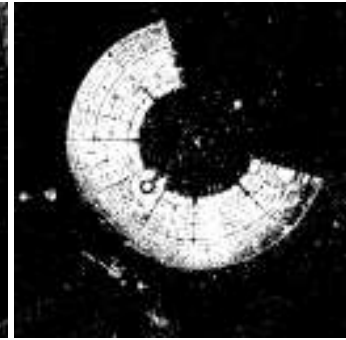

(g)

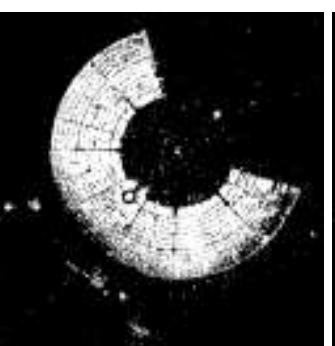

(1)

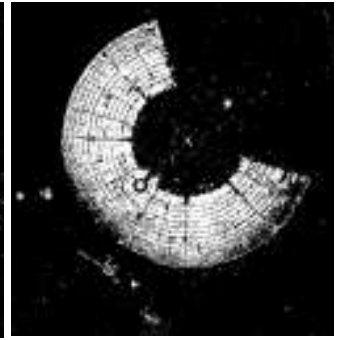

(h)

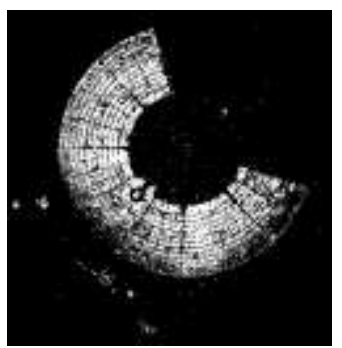

(m)

Fig. 5: Experimental results of TerraSAR-X dataset (a) Original image1 (b) Original image2 (c) Ground Truth (d) MR (e) LR (f) WFML (g) WFMLO (h) LPFMLO (i) AD (j) FD (k) WFAX (1) WFAXO (m) LPFAXO

Table 4: Quality Measures for Change Detection Description

$\left(\mathrm{F}_{\mathrm{P}}\right)$ False Positive : Unchanged pixels are erroneously classified as Changed pixels. $F_{P}=\sum_{i=1}^{n} f_{p}$

$\left(F_{N}\right)$ False Negative: Changed pixels are erroneously classified as Unchanged pixels. $F_{N}=\sum_{i=1}^{m} f_{n}$

$\left(\mathrm{T}_{\mathrm{P}}\right)$ True Positive: Change pixels are correctly classified as Change pixels. $T_{P}=\sum_{i=1}^{n} t_{p}$

$\left(\mathrm{T}_{\mathrm{N}}\right)$ True Negative: Change pixels are wrongly classified as Unchanged pixels.

(FAR) False Alarm Rate: The false discovery rate depends on the value of $F_{P}$ and $T_{P}$. $T_{N}=\sum_{i=1}^{m} t_{i}$ $F A R=\frac{F_{P}}{F_{P}+T_{N}}$ $E_{R}=\frac{O_{E}}{N}$

$\left(E_{R}\right)$ Error Rate: The ratio between Overall Error $\left(\mathrm{O}_{E}\right)$ to the total number of pixels $(N)$. 


\begin{tabular}{|c|c|}
\hline $\begin{array}{l}\left(\mathrm{K}_{\mathrm{C}}\right) \text { Kappa Coefficient: It measures the classification between change and error pixels. KC is a maximum to one for per- } \\
\text { fect classified image. } \\
\qquad P_{R E}=\frac{\left(T_{P}+F_{P}\right) \cdot\left(F_{N}+T_{P}\right)+\left(F_{N}+T_{N}\right) \cdot\left(F_{P}+T_{N}\right)}{N^{2}} \\
\text { where } \mathrm{N} \text { is a number of the entire pixels. }\end{array}$ & $K_{C}=\frac{P_{C C}-P_{R E}}{1-P_{R E}}$ \\
\hline (P) Precision: This is termed as precision, is ratio of changed pixels are correctly classified as changed. & $P=\frac{T_{P}}{F_{P}+T_{P}}$ \\
\hline$\left(\mathrm{P}_{\mathrm{CC}}\right)$ Percentage Correct Classification : The accuracy of prediction, state the correct classification of the result. & $P_{C C}=\frac{T_{P}+T_{N}}{N}$ \\
\hline (R) Recall: The ratio of changed pixels are classified as unchanged. It is also termed as Recall. & $R=\frac{T_{P}}{T_{P}+F_{N}}$ \\
\hline $\mathrm{F}_{1}$ Score: It measures the harmonic mean of the true positive rate and positive predictive value. & $F_{1}=\frac{2 T_{P}}{2 T_{P}+F_{P}+F_{N}}$ \\
\hline $\begin{array}{l}\left(\mathrm{O}_{\mathrm{E}}\right) \text { Overall Error: The sum of unchanged pixels is incorrectly classified into changed pixels and changed pixels are incor- } \\
\text { rectly classified into unchanged pixels. }\end{array}$ & $O_{E}=F_{P}+F_{N}$ \\
\hline
\end{tabular}

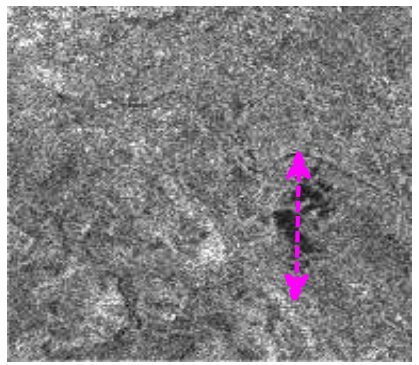

(b)
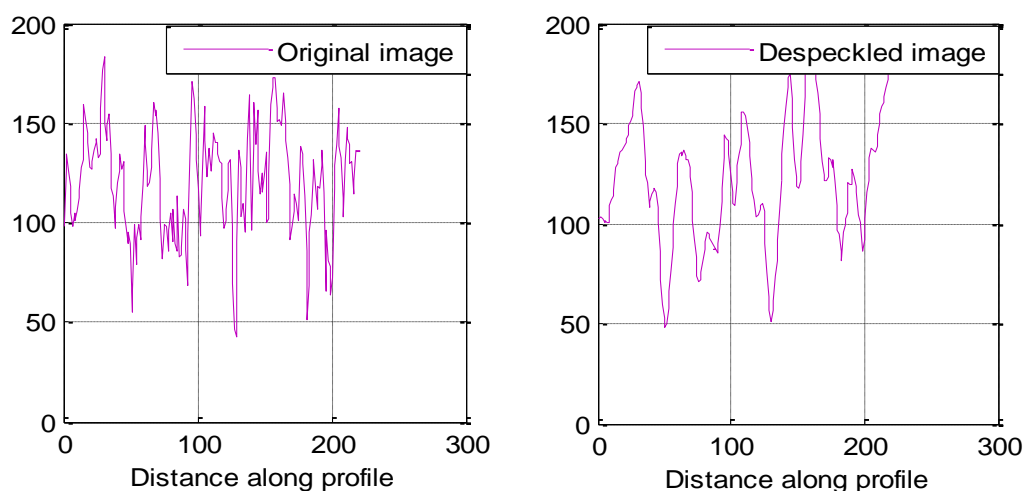

Fig. 6: Experimental results of image profile (b) ERS-2 dataset with marginal on $220^{\text {th }}$ column, Distance along profile on original and despeckled image



(a)

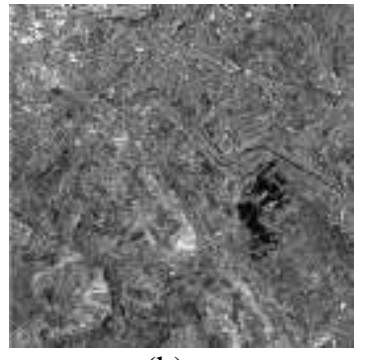

(b)

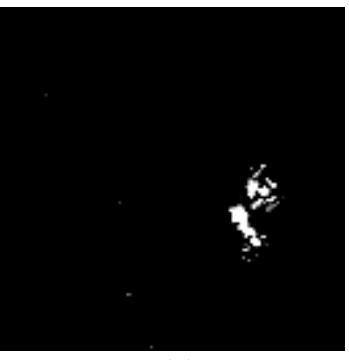

(c)



(d)



(i)

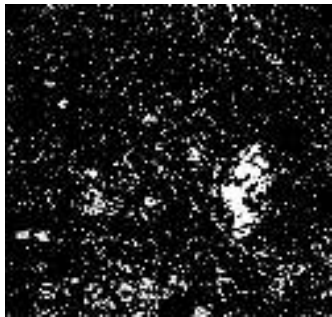

(e)

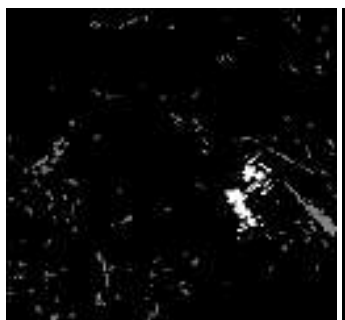

(j)

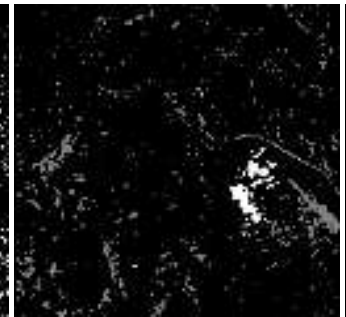

(f)



(k)

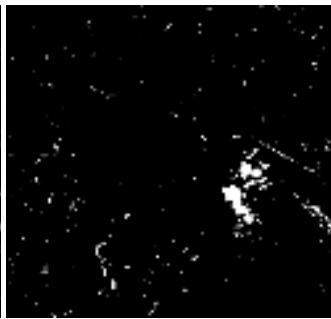

(g)

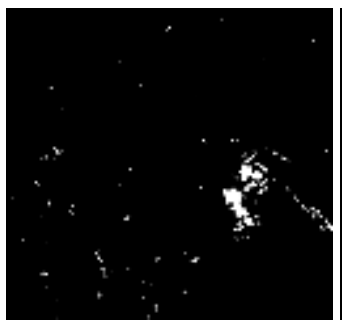

(1)



(h)

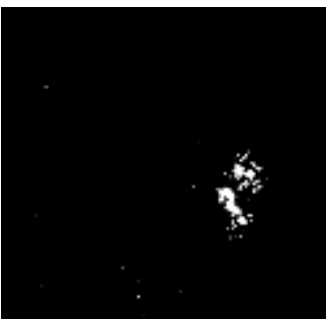

(m)

Fig. 7: Experimental results of ERS-2 dataset (a) Original image1 (b) Original image2 (c) Ground Truth (d) MR (e) LR (f) WFML (g) WFMLO (h) LPFMLO (i) AD (j) FD (k) WFAX (l) WFAXO (m) LPFAXO 
Table 5: Performance Evaluation for ERS-2 dataset

\begin{tabular}{|c|c|c|c|c|c|c|c|c|c|c|c|c|}
\hline Methods & $F_{P}$ & $\mathrm{~F}_{\mathrm{N}}$ & $\mathrm{T}_{\mathrm{P}}$ & $\mathrm{T}_{\mathrm{N}}$ & $\mathrm{P}_{\mathrm{CC}}$ & FAR & $F_{1}$ & $\mathrm{O}_{\mathrm{E}}$ & $E_{R}$ & $\mathrm{~K}_{\mathrm{C}}$ & $\mathrm{P}$ & $\mathrm{G}$ \\
\hline MR & 11181 & 70 & 1523 & 72481 & 0.868 & 0.1336 & 0.2131 & 11251 & 0.132 & 0.186 & 0.1199 & 0.3385 \\
\hline LR & 412 & 472 & 1096 & 83275 & 0.9896 & 0.0049 & 0.7126 & 884 & 0.0104 & 0.7073 & 0.7268 & 0.7127 \\
\hline WFML & 197 & 555 & 893 & 83610 & 0.9912 & 0.0024 & 0.7037 & 752 & 0.0088 & 0.6993 & 0.8193 & 0.7108 \\
\hline ADI & 777 & 298 & 301 & 83879 & 0.9874 & 0.0092 & 0.359 & 1075 & 0.0126 & 0.3531 & 0.2792 & 0.3746 \\
\hline FDI & 558 & 235 & 493 & 83969 & 0.9907 & 0.0066 & 0.5542 & 793 & 0.0093 & 0.5497 & 0.4691 & 0.5636 \\
\hline WFAX & 180 & 516 & 665 & 83856 & 0.9918 & 0.0021 & 0.6565 & 696 & 0.0082 & 0.6524 & 0.787 & 0.6657 \\
\hline WFAXO & 154 & 468 & 907 & 83726 & 0.9927 & 0.0018 & 0.7447 & 622 & 0.0073 & 0.741 & 0.8549 & 0.7509 \\
\hline
\end{tabular}
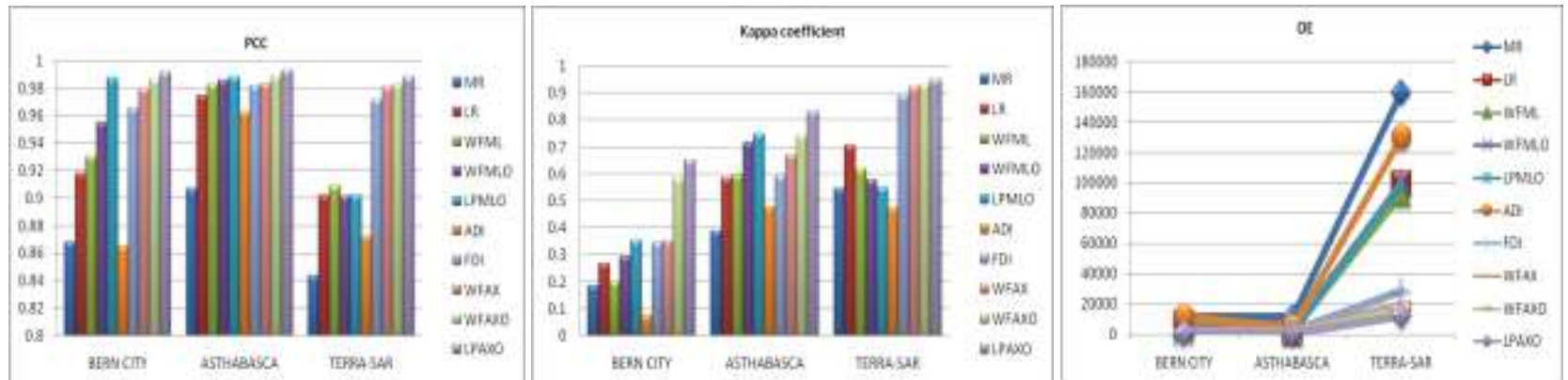

Fig. 8: Performance Evaluation for various datasets (a) $\mathrm{P}_{C C}$ (b) $\mathrm{K}_{C}$ (c) $\mathrm{O}_{\mathrm{E}}$

\section{Results and Discussion}

\subsection{Dataset Details}

In this study, three datasets of SAR images are performed to evaluate the integrity method of speckle reduction and change detection process.

I. The First dataset is acquired by Envisat satellite over the comparative study period between May 2000 and 2005 in Asthabasca River, Canada. It shows large amount of oil sands deposited over the river. The minimum range of 285 x $426 \mathrm{im}-$ ages is preferred for analyzing change detection application.

II. The Second dataset is TerraSAR-X images acquired in black rock city, Nevada, USA. The study period is to analyze the structure of before and after burning man festival of 2011 . The size of the images is $2436 \times 2000$.

III. The Third dataset is acquired by ERS-2 satellite taken from the Aare valley in between Bern and Thun cities, Switzerland. This dataset is acquired to realize the study of flood formation around in April 1999 and May 1999. The size of 295 x 289 is utilized for change detection application.

\subsection{Quality Measures}

This session used to validate the metric parameters of change detection analysis. The above datasets are evaluated using various confusion matrix parameters [1] are listed in Table 4.

\subsection{Results on Various Datasets}

The proposed change detection method is implemented and compared with existing methods for three different multitemporal SAR images. The effectiveness of the proposed method is carried out by following phases: preprocessing, change detection and performance metrics. In the first stage, preprocessing is used to remove speckle noise to improve the picture quality for further post processing applications. The Laplacian pyramid based SRAD method is carried out for excellent noise reduction. An image profile has shown the effectiveness of distance along with the profile for speckle and despeckled images for various datasets are shown in Fig. 2, Fig. 4 and Fig. 6 respectively. The second stage is the main objective of change detection by Absolute difference (AD) and Foreground detection (FD) techniques. The $\mathrm{AD}$ is the magnitude difference between two multitemporal SAR images and FD is a logical XOR operation between two images. The performance of FD using the XOR operator predicts the exact changes of both images. The multiscale fusion method is applied to AD and FD images. Laplacian pyramid based fusion approach is used to fuse primary resultant images of AD and FD (LPFAX). Then wavelet fusion coefficient also applied to fuse resultant images of $\mathrm{AD}$ and FD (WFAX) to compare the performance.

In this study, the ratio operators of Log ratio (LR), Mean ratio (MR) methods are used with and without fusion methods. The mean ratio method depends on local mean intensity value of the difference image. The log ratio operator does not produce exact change region because ratio method is abate the high intensity pixels. The ratio methods are fused by Laplacian (LPFML) and wavelet pyramid coefficients (WFML), even though the fused image also fades the background information. On the other hand, the proposed techniques of $\mathrm{AD}$ and FD are performed by with and without fusion technique. The LPFAX is decomposing the both images and used the separate fusion rule on band pass layers and low pass layer. The fusion rule of merged standard deviation and entropy is used for low pass image and fusion rule of maximum local area energy is used for band pass images, which will enable the fusion to highlight the edges and boundary regions. The wavelet fusion based AD and FD (WFAX) also examined to compare the pyramid fusion method. Then, the Otsu thresholding algorithm is adapted finally to segment perfect change detection. By this segmentation method, optimized threshold value is evaluated by maximum variance of between class variance. Finally, the perform gives better results in WFAXO comparable than WFMLO. The image WFAXO gives perfect and correct classified result without any unwanted dark spots. The experiment of various methods is shown in Fig. 3 for an Envisat dataset, Fig. 5 for TerraSAR-X dataset, and Fig. 7 for ERS-2 dataset. The quality measures are evaluated to prove the effectiveness of speckle reduction and tabulated in Table 1. The proposed change detection results are compared by various parameters in Table 4 and the performance measures are tabulated in Table 2 for Envisat dataset, Table 3 for TerraSAR-X dataset and Table 5 for ERS- 2 dataset. The comparative study of quality measures is plotted to visualize the performance for various datasets shown in Fig. 8 - Fig. 11. These quality measures exposes that the proposed method excellent result compare than existing methods, the proposed method achieves better performance result. 


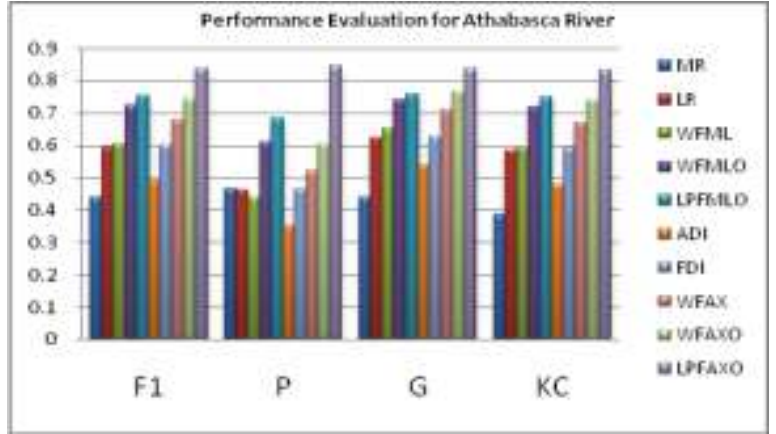

Fig. 9: Performance Evaluation for Envisat dataset

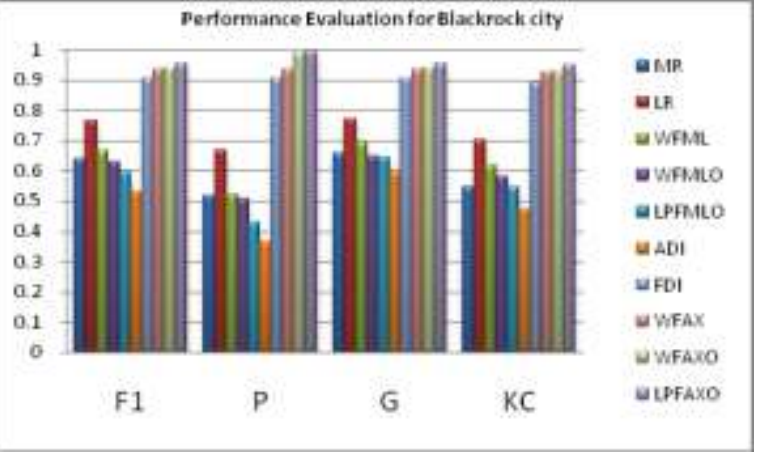

Fig. 10: Performance Evaluation for TerraSAR-X dataset



Fig. 11: Performance Evaluation for ERS-2 dataset

\section{Conclusion}

In this paper, we propose a unique fusion based change detection method for Synthetic Aperture Radar (SAR) imagery by Otsu's thresholding. This study incorporates speckle noise removal and change detection of multitemporal SAR images. The despeckling technique of Laplacian pyramids based speckle reducing anisotropic diffusion (LPSRAD) approach is used. The multitemporal SAR images are involved with Absolute difference and Foreground detection methods. The primary difference images are illustrated with multiscale fusion rule to improve the individual quality. Finally the automatic Otsu's threshold algorithm is used to attain a perfect change image. The results are evaluated by high quality measures and proved the proposed method shows better performance than the existing methods.

\section{References}

[1]AnishaM.lal, Margret anouncia, "Semi-supervised change detection approach combining sparse fusion and constrained k-means for multitemporal remote sensing images". The Egyptian journal of remote sensing and space sciences. 18, 279-288. (2015).

[2]F Zhang, Y M Yoo, L M Koh and Y Kim, "Nonlinear diffusion in laplacian pyramid domain for ultrasonic speckle reduction". IEEE Trans. on Medical imaging, Vol. 26, No.2, 200-211.(2007).
[3]L.Bruzzone, D.F. Prieto, "Automatic analysis of the difference image for unsupervised change detection". IEEE Transactions of Geo science and remote sensing. Vol. 38, no. 3. 1171-1182 (2000).

[4]Maoguo, Yu Li, Lichengjiao, Linzhi, "SAR change detection based on intensity and texture changes". ISPRS journal of photogrammetry and remote sensing. 93, 123-135. (2014).

[5]Maoguo, Zhiqiangzhou, jingjing, "Change detection in Synthetic aperture radar images based on image fusion and fuzzy clustering". IEEE Transactions on image processing. 21, 2141-2151. (2012).

[6]Nobuyuki Otsu, A., "Threshold selection method from gray level histograms", IEEE Trans. on systems, man and cybernetics, Vol. SMC-9, no. 1, 62-66. (1979).

[7]Peter J. Burt, Edward H. Adalson, "The Laplacian pyramid as a compact image code". IEEE Trans. on communications. Vol. COM-31, no.4, 532-540.(1983).

[8]R.Vijayageetha, S.Kalaivani, "Laplacian pyramid based speckle reducing anisotropic diffusion (LPSRAD) for SAR images". International Journal of Applied Engineering Research (IJAER), ISSN 0973-4562 Vol. 10, No.30 (2015)

[9]Yu and Acton, "Speckle Reducing Anisotropic Diffusion". IEEE Transactions on Image processing, Vol. 11, no. 11, 1260-1270. (2002).

[10]Y.Ban, O. A. Yousif, "Multitemporal Space SAR data for urban change detection in china". IEEE Journal. of selected topics in applied earth observations and remote sensing, Vol. 5, No. 4, 1087 1094. (2012). 